\title{
Class-modeling analysis reveals T-cell homeostasis disturbances involved in loss of immune control in elite controllers
}

\author{
José M. Benito ${ }^{1,2^{*}}$, María C. Ortiz ${ }^{3}$, Agathe León ${ }^{4}$, Luis A. Sarabia ${ }^{3}$, José M. Ligos ${ }^{5}$, María Montoya ${ }^{5}$, Marcial Garcia ${ }^{1,2}$, \\ Ezequiel Ruiz-Mateos ${ }^{6}$, Rosario Palacios ${ }^{7}$, Alfonso Cabello ${ }^{8}$, Clara Restrepo ${ }^{1,2}$, Carmen Rodriguez ${ }^{9}$, \\ Jorge del Romero ${ }^{9}$, Manuel Leal ${ }^{6}$, María A. Muñoz-Fernández ${ }^{10}$, José Alcamí ${ }^{11}$, Felipe García ${ }^{4}$, Miguel Górgolas ${ }^{8}$, \\ Norma Rallón $n^{1,2^{*}}$ and On behalf of ECRIS integrated in the Spanish AIDS Research Network
}

\begin{abstract}
Background: Despite long-lasting HIV replication control, a significant proportion of elite controller (EC) patients may experience CD4 T-cell loss. Discovering perturbations in immunological parameters could help our understanding of the mechanisms that may be operating in those patients experiencing loss of immunological control.

Methods: A case-control study was performed to evaluate if alterations in different T-cell homeostatic parameters can predict CD4 T-cell loss in ECs by comparing data from EC patients showing significant CD4 decline (cases) and EC patients showing stable CD4 counts (controls). The partial least-squares-class modeling (PLS-CM) statistical methodology was employed to discriminate between the two groups of patients, and as a predictive model.

Results: Herein, we show that among T-cell homeostatic alterations, lower levels of naïve and recent thymic emigrant subsets of CD8 cells and higher levels of effector and senescent subsets of CD8 cells as well as higher levels of exhaustion of CD4 cells, measured prior to CD4 T-cell loss, predict the loss of immunological control.

Conclusions: These data indicate that the parameters of T-cell homeostasis may identify those EC patients with a higher proclivity to CD4 T-cell loss. Our results may open new avenues for understanding the mechanisms underlying immunological progression despite HIV replication control, and eventually, for finding a functional cure through immune-based clinical trials.
\end{abstract}

Keywords: Elite controllers, CD4 T-cell loss, Class modeling, T-cell homeostatic parameters, CD8 exhaustion

\section{Background}

Human immunodeficiency virus-1 (HIV-1) infection is mostly characterized by ongoing viral replication leading to a progressive decline of CD4+ T cells and eventually progression to clinical AIDS. Nevertheless, there is a small subset of HIV-1+ subjects who are able to spontaneously control HIV-RNA replication in plasma to levels below the limit of detection in the absence of

\footnotetext{
* Correspondence: jbenito1@hotmail.com;

jose.benito@hospitalreyjuancarlos.es; normaibon@yahoo.com; norma.rallon@hospitalreyjuancarlos.es

'IIS-Fundación Jiménez Díaz, UAM, Av. Reyes Católicos, 2, 28040 Madrid, Spain

Full list of author information is available at the end of the article
}

antiretroviral therapy. They are called elite controllers (ECs) and represent less than $1 \%$ of the total of HIVinfected patients [1]. ECs have been considered as a model of spontaneous functional cure and the role of host genetic factors, innate and adaptive immune responses, viral characteristics, and their interactions have been analyzed in several studies [1-5].

However, ECs constitute a heterogeneous group in terms of genetic and immunologic characteristics [6], and no single mechanism has been described as being responsible for controlling viral replication [5-7]. These patients are also heterogeneous from the clinical point of view, since some of them show either virologic and/or immunologic progression [8-11]. Studies with large 
cohorts of EC patients have reported that as many as $25 \%$ of them experience virological progression (losing the ability to maintain HIV replication control) [11], and around $20 \%$ experience immunological progression (a decline in CD4 counts) even in the absence of virological progression $[8,9]$, as we have also previously demonstrated in our cohort of HIV controllers of the Spanish AIDS Research Network (the ECRIS cohort) [11]. However, the mechanisms accounting for this CD4 T-cell loss in ECs remain largely unknown.

Given the relevance of different alterations of T-cell homeostasis in the CD4 depletion observed in HIV patients with uncontrolled viral replication [12-14], some of these same mechanisms might be operating in EC patients experiencing CD4 T-cell loss. In this regard, previous studies have shown that even though EC patients are able to suppress HIV replication to an undetectable level, they present different T-cell homeostasis disturbances compared to uninfected controls and/or to HIV non-controllers with suppression of HIV replication mediated by combination antiretroviral therapy (cART) [15-20], suggesting that Tcell homeostasis disturbances are still operating in EC patients and could potentially be involved in the immunological progression.

To test this hypothesis, and with the final aim of finding predictors of immunological progression, herein we have comprehensively compared several important parameters of T-cell homeostasis in EC patients with long-lasting HIV replication control but experiencing immunological progression with those for EC patients showing no progression. The partial least-squares-class modeling (PLS-CM) methodology was applied as a statistical modeling technique to discriminate between the two groups of patients, and as a predictive model.

\section{Methods}

\section{Study population}

This is a case-control study including adult patients with chronic HIV infection and with long-term spontaneous control of HIV replication (EC patients) during the whole study follow-up period, recruited from the cohort of HIV controllers of the Spanish AIDS Research Network (ECRIS cohort) launched in 2013. ECRIS is an open multicenter cohort of HIV controller patients. The data are from the Spanish Long-Term Non-Progressors (LTNP) cohort and the Spanish AIDS Research Network (CoRIS) cohort [21], and from different clinical centers in Spain. To be included in the ECRIS cohort, EC patients were defined as asymptomatic individuals with at least three consecutive plasma HIV viral load (pVL) determinations below the detection limit ( $\mathrm{pVL}<50$ copies/mL) during at least 12 months of follow-up, in the absence of any cART. The characteristics of the ECRIS cohort have been described in detail elsewhere
[11]. Only those EC subjects maintaining long-term control of HIV replication (more than 3 years) with regular immunovirological follow-ups (CD4 counts and pVL) and with available baseline cryopreserved cellular samples were included in the study. Baseline was the beginning of the follow-up period as an EC patient.

\section{Selection of EC study groups}

The relevant CD4 data for each patient were measured during the follow-up period if the patient maintained their EC status. All of these data were used in a linear regression analysis to estimate the CD4 slope for the patient. According to the CD4 slope, the study population was categorized into two groups: EC controls, patients with stable CD4 counts during the follow-up period (CD4 count slope not significantly different from zero) and EC cases, patients whose CD4 counts declined during the follow-up period (a statistically significant and negative CD4 count slope). A total of $36 \mathrm{EC}$ patients were included: 22 being EC controls and 14 being EC cases (Additional file 1 and Fig. 1). Moreover, 14 ageand sex-matched healthy controls (HIV-uninfected individuals) were included as a healthy control group (HCs).

\section{Cell samples}

Anonymized samples from both groups of patients were kindly provided by the HIV BioBank, which is integrated into the Spanish AIDS Research Network (RIS) [22]. Anonymized data were taken from the ECRIS cohort database (see Additional file 1), according to the sample assignment agreement reference 19/15 dated 25 August 2015. All analyses were done with cryopreserved peripheral blood mononuclear cells (PBMCs), and were performed when both groups of EC patients (cases and controls) presented similar levels of CD4 counts, that is prior to CD4 T-cell loss in EC cases. EDTA-anticoagulated blood was obtained by venipuncture. PBMCs were immediately isolated by density gradient centrifugation using Ficoll-Hypaque (Sigma Chemical Co., St. Louis, MO), frozen in fetal calf serum plus $10 \%$ dimethyl sulfoxide, and stored in liquid nitrogen in the Spanish AIDS Research Network HIV BioBank. The viability of thawed PBMCs was always greater than $85 \%$.

\section{Immunophenotypic analysis}

We used a comprehensive approach of simultaneously measuring different T-cell parameters involved in HIV pathogenesis: differentiation stage; recent thymic emigrants (RTEs); activation, exhaustion, and apoptosis of $\mathrm{T}$ memory stem cells (Tscm cells); T regulatory cells (Treg cells); replicative senescence; and response to homeostatic cytokines. We selected a total of 16 different monoclonal antibodies distributed in two different panels of 11 and 13 antibodies (plus a cell viability marker), which in combination defined 350 different 

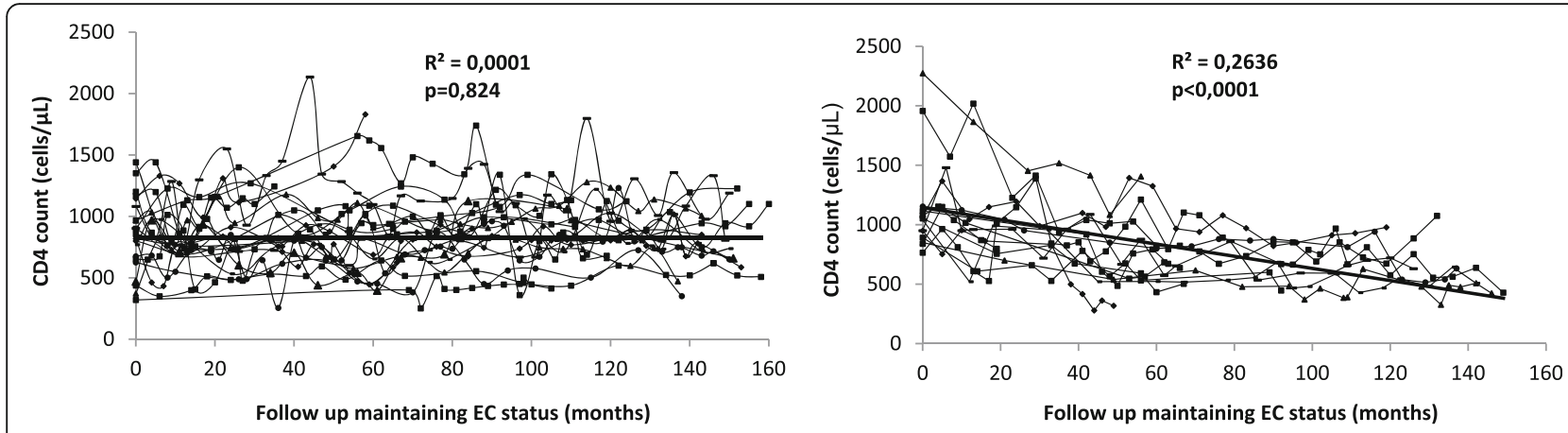

Fig. 1 Scatterplots showing the evolution of the CD4 count during the follow-up period for two groups of EC patients: those showing stable CD4 counts (left) and those showing a significant CD4 decline (right). Each individual line represents data from one patient. EC elite controller

unique T-cell subsets that were analyzed by multiparametric flow cytometry. A complete list of all monoclonal antibodies and fluorochromes used in the study as well as antibodies included in each of the two staining panels is shown in Additional file 2. A minimum of 2 million of PBMCs from each patient were stained with each antibody panel, and a detailed description of staining conditions and definitions of T-cell subsets analyzed is given in Additional file 3.

\section{Statistical analyses}

The main characteristics of the study population and the different parameters evaluated are expressed as median and interquartile range. Comparisons between groups were done using a Mann-Whitney $U$ test. The twotailed $p$ values were considered as significant only when they were lower than 0.05 . All statistical analyses were performed using the SPSS software version 15 (SPSS Inc., Chicago, IL, USA). PLS-CM was employed to discriminate between the different groups of subjects included in the study (control patients, case patients, and HCs). Two PLS models were generated: one to discriminate between EC patients and HCs (PLS-1) and another to discriminate between cases and control groups of EC patients (PLS-2). In these models, $350 \mathrm{im}$ munological parameters were used as predictor (explicative) variables of the model, and the subjects' group was the predicted (response) binary variable. For PLS-1, a value of 0 was assigned to EC patients and a value of 1 was assigned to HCs. For PLS-2, a value of 0 was assigned to EC controls and a value of 1 to EC cases.

In the first step, PLS was used to select a minimum set of predictor variable to reduce the original $350 \mathrm{im}$ munological variables to simplify the analysis without losing any information. The parameter employed to make this selection, called variable influence on projection (VIP), summarizes the importance of the predictor variable on the model taking into account the amount of explained variance of the predicted (response) variable.
In the second step, a PLS model was built using the selected set of predictor variables grouped together to generate a minimum set of latent variables (LVs) that are not directly observed (measured) but rather inferred (through a mathematical model) from other variables that are observed (directly measured). Each LV is a linear combination of the original set of observable variables. A clear advantage of using LVs is that it reduces the dimensionality of data. The number of LVs included in the PLS model is optimized to maximize the explained variance of the $X$ (predictor variables) and $Y$ (response variable) components of the model. Applying this PLS model, a predicted value for the response variable was estimated for each sample and for these predicted values, a probability distribution was generated. For this distribution, the probabilities $\alpha$ and $\beta$ for type I and II errors of the hypothesis test, respectively, were calculated. The sensitivity $[(1-\alpha) \times 100]$ and specificity $[(1-\beta) \times 100]$ of the PLS model were used to assign each sample to its correct group. A detailed explanation of PLS-CM is given in Additional file 4.

\section{Results}

\section{Patient characteristics}

The CD4 slope during the follow-up period for controls and cases is shown in Table 1 . Of the 36 EC patients included in the study, 22 were considered controls (stable CD4 counts during the follow-up period) and 14 were considered cases (significant CD4 decline during the follow-up period). All immunological parameters were measured prior to CD4 T-cell loss in EC cases. The evolution of CD4 counts during the follow-up period for controls and cases is shown in Fig. 1. The main characteristics of the controls and cases are shown in Table 1. There were no significant differences between control and case groups in terms of age, median time since diagnosis, CD4 count at the beginning of follow-up, CD4 count at the commencement of the study, percentage of patients infected with hepatitis $\mathrm{C}$ virus, percentage of 
Table 1 Characteristics of patients included in the study

\begin{tabular}{|c|c|c|c|}
\hline Characteristic & Control group $(n=22)$ & Case group $(n=14)$ & $p$ value \\
\hline$\overline{\text { Age }(\text { years) }}{ }^{\mathrm{a}}$ & $40[35,48]$ & $42[39,48]$ & 0.58 \\
\hline Gender (\% of males) & 33 & 69 & 0.04 \\
\hline Follow-up maintaining EC status (years) & $12[10,13]$ & $9[5,12]$ & 0.02 \\
\hline Time since HIV diagnosis (years) & $19.3[14.8,24.1]$ & $19.3[9.6,21.8]$ & 0.62 \\
\hline CD4 count at the beginning of follow-up (cells/ $\mu \mathrm{L}$ ) & $838[646,1097]$ & $1047[881,1125]$ & 0.06 \\
\hline CD4 count at the end of follow-up (cells/ $\mu \mathrm{L}$ ) & $931[675,1147]$ & $632[443,843]$ & 0.01 \\
\hline CD4 slope (cells/uL per year) & $8.9[1.2,19]$ & $-66[-113,-32]$ & $<0.0001$ \\
\hline CD4 count at the moment of the study (cells/ $\mu \mathrm{L}$ ) & $889[752,1051]$ & $886[548,1280]$ & 0.87 \\
\hline Hepatitis C virus positive (\%) & 80 & 69 & 0.68 \\
\hline Patients with non-AIDS defining events during the follow-up period (\%) & 11 & 11 & 1 \\
\hline Number of HIV-RNA blips ( $p V L$ > 50 copies/mL) & $2[0,2.3]$ & $1[0,2.3]$ & 0.43 \\
\hline
\end{tabular}

EC elite controller

${ }^{a}$ Data for continuous variables are given as median [interquartile range]

patients presenting with non-AIDS defining events during the follow-up period, and number of HIV-RNA blips during the follow-up period.

\section{PLS class modeling of EC patients versus $\mathrm{HC}$ subjects (PLS-1)}

First a PLS model was generated to discriminate between EC patients and HIV-seronegative HC subjects. A data matrix with 50 samples (36 EC patients and 14 $\mathrm{HCs}$ ) and 350 variables was used to fit a first PLS model without any data preprocessing. A binary variable took the value 0 if the sample was from an EC patient and 1 if it was from a HC. This model let us choose 93 variables with VIP values higher than 1 so that we could simplify the analysis without losing any information. A new PLS model (PLS-1) with the same criteria and a reduced data matrix with 93 variables was built. Three LVs were included in the final PLS model, and the percentage of explained variance of the response variable $(y)$ and predictor variables $(X)$ is shown in Table 2 for PLS1. A normal distribution for each class (EC patients and $\mathrm{HCs}$ ) was fitted with the values of the response variable calculated by the PLS model because the $p$ values of the normality tests were greater than 0.1 . These density functions for each class are plotted in Fig. 2. The mean and standard deviation of the calculated response variable for the PLS-1 model for the EC patient and $\mathrm{HC}$ classes are, respectively, $0.075 \pm 0.219$ and $0.810 \pm 0.146$ (Fig. 2).

By choosing a threshold to differentiate between EC patients and $\mathrm{HC}$ subjects, we can see in Fig. 2 that the small area under the density function of the class of EC patients on the right of the threshold is the probability $\alpha$ of rejecting the null hypothesis $\left(\mathrm{H}_{0}\right.$ : the sample belongs to the EC patient class) when it is true. On the other hand, the small area under the density function of the class of HCs on the left of the threshold is the probability $\beta$ of accepting the null hypothesis $\left(\mathrm{H}_{0}\right)$ when it is false. By changing the threshold, a risk curve of type $\alpha$ error versus type $\beta$ error can be generated and from this we can estimate the pair of equal values of sensitivity $[(1-\alpha) \times 100]$ and specificity $[(1-\beta) \times 100]$ of the model (Fig. 3). For the PLS-1 model, these values were both $98 \%$. To visualize the ability of the PLS model to discriminate between EC patients and HCs, Fig. 4 (left) shows the projection in a plane of the scores for LV2 and LV3 (rotated $25^{\circ}$ counterclockwise) for each of the 50 individuals of the study (36 EC patients and $14 \mathrm{HCs}$ ).

Table 2 Percentage of variance of predictor ( $X$ block) and response ( $y$ block) variables explained by the PLS models. Individual (partial) and accumulated (total) explained variance for each latent variable of the models are shown

\begin{tabular}{|c|c|c|c|c|c|c|c|c|c|}
\hline \multirow{3}{*}{$\begin{array}{l}\text { Latent } \\
\text { variable }\end{array}$} & \multicolumn{4}{|l|}{ PLS-1 } & \multirow{3}{*}{$\begin{array}{l}\text { Latent } \\
\text { variable }\end{array}$} & \multicolumn{4}{|l|}{ PLS-2 } \\
\hline & \multicolumn{2}{|c|}{$x$ block } & \multicolumn{2}{|c|}{ y block } & & \multicolumn{2}{|c|}{$x$ block } & \multicolumn{2}{|c|}{ y block } \\
\hline & Partial & Total & Partial & Total & & Partial & Total & Partial & Total \\
\hline 1 & 91.4 & 91.4 & 31.6 & 31.6 & 1 & 92.1 & 92.1 & 38.1 & 38.1 \\
\hline 2 & 1.4 & 92.8 & 43.3 & 74.9 & 2 & 1.4 & 93.5 & 26.2 & 64.3 \\
\hline \multirow[t]{2}{*}{3} & 1.1 & 93.9 & 6.1 & 81.0 & 3 & 0.8 & 94.3 & 16.9 & 81.2 \\
\hline & & & & & 4 & 0.7 & 95.0 & 3.4 & 84.6 \\
\hline
\end{tabular}



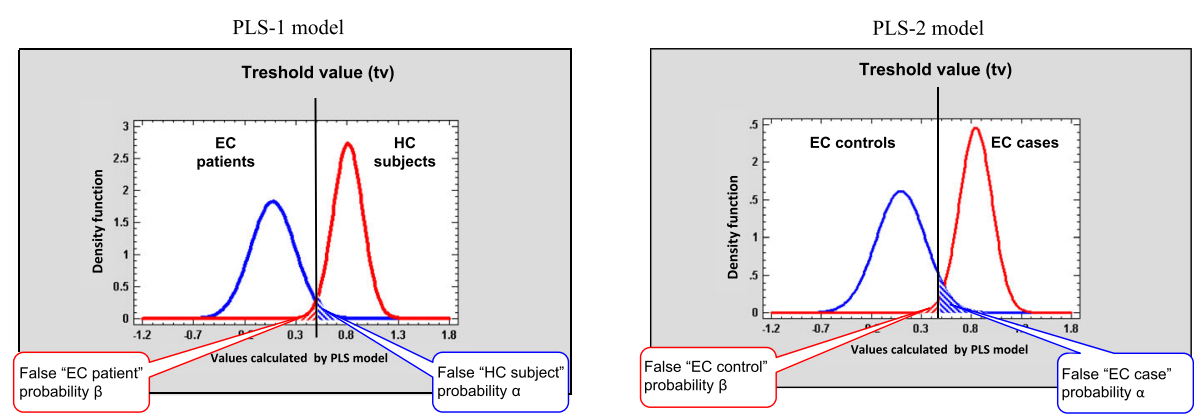

Fig. 2 Density functions for values calculated by models PLS-1 (left) and PLS-2 (right) for the two classes of individuals: EC patients and HC subjects for the PLS-1 model, and EC cases and EC controls for the PLS-2 model. The vertical line represents a threshold value chosen to differentiate between the classes. The small overlapping areas to the right and to the left of this line represent the probabilities a and $\beta$, respectively. EC elite controller, HC healthy control

This plot has a vertical line (a rotated LV2 value) that perfectly separates EC patients (on the left) and HC subjects (on the right).

PLS class modeling of EC cases versus EC controls (PLS-2) PLS class modeling was applied to discriminate between EC cases (EC patients showing a CD4 decline during the follow-up period) and EC controls (EC patients with stable CD4 counts during the follow-up period). Firstly, by fitting a PLS model with samples from 36 patients (22 EC controls and 14 EC cases), 77 variables from the 350 original ones with a VIP value higher than 1 were selected. No data preprocessing was used and a binary response variable took the value 0 if the sample was from an EC control and the value 1 if it was from an EC case. The same procedure as used for PLS-1 was followed.

A PLS regression (PLS-2) with the same previous criteria and the reduced data matrix with 77 variables was built with four LVs. The percentages of explained variance by the model of the $X$ and $y$ variables are shown in Table 2 (under PLS-2). We checked that the $p$ values of the normality tests were greater than 0.10 and therefore, a normal distribution for each class (EC cases and EC controls) was fitted with the values of the response variable calculated by the PLS model. The normal density functions for each class were plotted (Fig. 2). The mean values and standard deviation of the response variable calculated by the PLS model for the EC case and EC control classes were respectively $0.096 \pm 0.247$ and 0.846 \pm 0.162 (Fig. 2).

By changing the threshold, a risk curve of type $\alpha$ versus type $\beta$ errors was generated and from this we estimated the pair of equal values of sensitivity $[(1-\alpha) \times 100]$ and specificity $[(1-\beta) \times 100]$ of the model (Fig. 3). For the PLS-2 model, these values were both $97 \%$. To visualize the ability of the PLS-2 model to discriminate between EC cases and EC controls, Fig. 4 shows the projection in a plane of the scores for LV2 and LV3 rotated $49^{\circ}$ counterclockwise. This plot shows has a vertical line (a rotated LV2 value) that perfectly separates EC controls (on the left) and EC cases (on the right).

The PLS model shows that the immunological variables have information with sufficient specificity and sensitivity to discriminate between the EC controls and the EC cases (this is also true for discriminating between

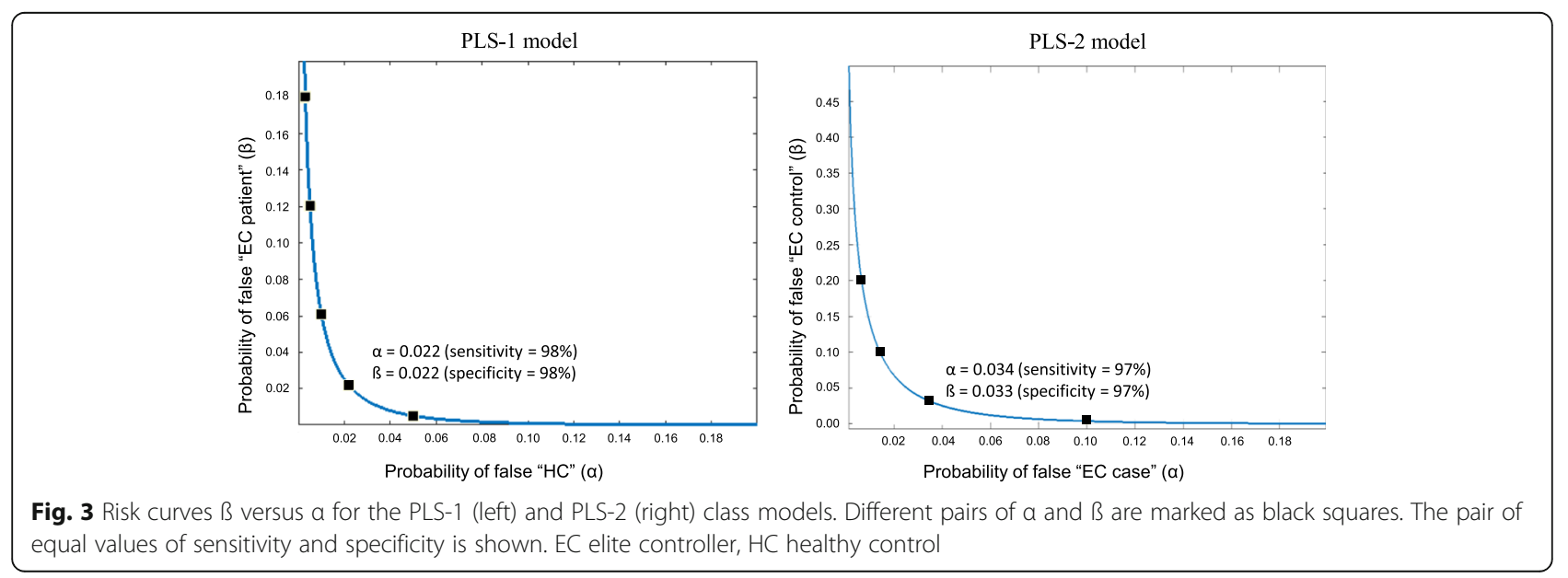



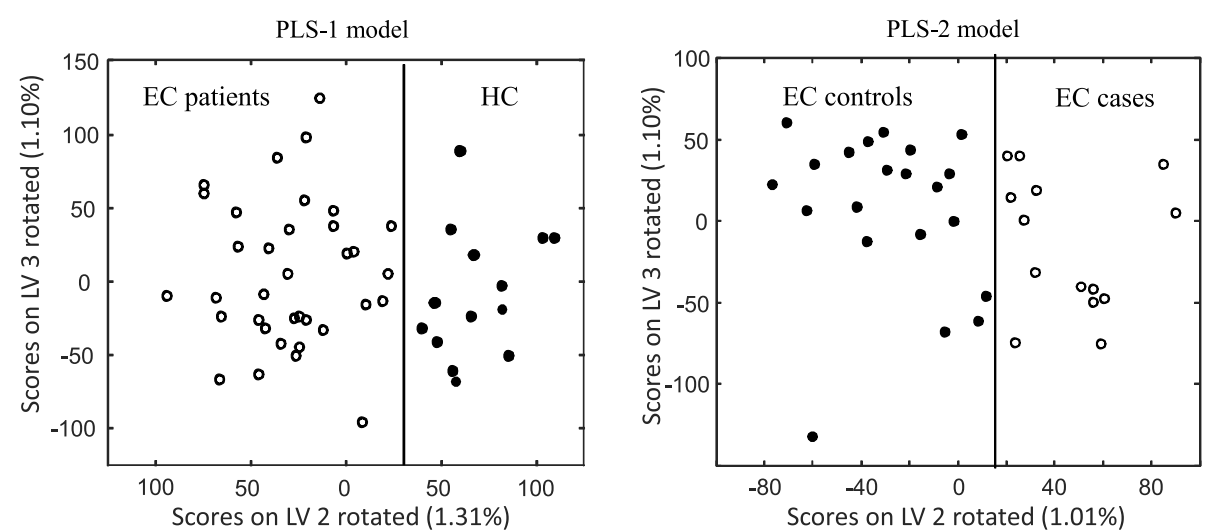

Fig. 4 Dot plots showing the projection of the scores for LV2 and LV3 for each subject included in the study. Left: Scores for EC patients (open circles) and HC subjects (black circles) obtained with the PLS-1 model. Right: Scores for EC controls (black circles) and EC cases (open circles) obtained with the PLS-2 model. EC elite controller, HC healthy control, LV latent variable

EC patients and $\mathrm{HC}$ subjects). There is an underlying structure responsible for this capacity of discrimination that is reducible to two LVs. However, the immunological variables are all on the same scale (percentage) and it is not appropriate to normalize them since the variables that are noise in the discrimination acquire the same magnitude as those truly responsible for it and may be incorrectly included in the model. The consequence of not normalizing the data is twofold: (1) one of the LVs is linked to the distance between the vector formed by the mean values of the predictor variables and the origin of coordinates and (2) the immunological variables with a smaller range may not be selected due to a numerical effect. In addition, PLS tends to eliminate highly correlated variables because they do not carry independent information, although their immunological meaning may be relevant and different. Consequently, choosing the immunological variables for which the difference between classes is perceived is done by evaluating the individual distribution of their values, as in the Mann-Whitney $U$ test.

\section{T-cell parameters significantly altered in EC patients with respect to healthy subjects}

Of the 93 immunological variables selected by the PLS-1 model to differentiate EC patients from healthy subjects, 43 presented statistically significant differences when EC patients and healthy subjects were compared with the Mann-Whitney test. Since the immunological variables are all on the same scale and it is not appropriate to normalize them, the PLS-CM model did not normalize the variables, and for this reason the immunological variables with a smaller range may not have been selected. Nevertheless, those variables could have some relevant information. Thus, using the Mann-Whitney $U$ test, it was possible to evaluate those variables significantly different between EC patients and healthy subjects but that were not selected by the PLS model. In fact, there were another 36 immunological variables significantly different between EC patients and healthy subjects that were not selected by the PLS model due to a numerical effect. A complete list of immunological variables with significant differences is shown in Additional file 5.

Figure 5 shows levels for the most relevant variables in EC patients and healthy subjects. Among CD4 T-cell subsets, EC patients showed lower levels of Tscm and Treg subsets and higher levels of the CD45RA-CD27-CCR7subset compared to healthy subjects. Interestingly, expression of activation marker CD38 (but not of HLADR) was also lower in total CD4 T cells and in naïve CD4 T cells of EC patients. In contrast, co-expression of activation markers CD38 and HLADR was higher in Tscm cells of EC patients. The proportion of naïve $\mathrm{CD} 4$ cells lacking expression of CD28 was also higher in EC patients.

Among CD8 T-cell subsets, EC patients showed lower levels of naïve RTE (naïve cells expressing CD31) and Tscm subsets compared to healthy subjects. However, co-expression of activation markers CD38 and HLADR in total CD8+ cells and in effector (CD45RA + CD27CCR7-) subset was higher in EC patients. Expression of CD95 in activated (either CD38+ or HLADR+) subsets of CD8 cells was also higher in EC patients. Lastly, the proportion of $\mathrm{CD} 8$ cells lacking $\mathrm{CD} 28$ expression was also higher in EC patients (Fig. 5).

\section{T-cell parameters associated with loss of immunological control in EC patients}

Of the 77 immunological variables selected by the PLS model to differentiate between EC cases (EC patients showing a significant $\mathrm{CD} 4$ count decline) and $\mathrm{EC}$ controls (EC patients with a stable CD4 count), 12 presented statistically significant differences between EC cases and EC controls using the Mann-Whitney test. Interestingly, all of them were variables with the highest coefficients (loadings) in the 


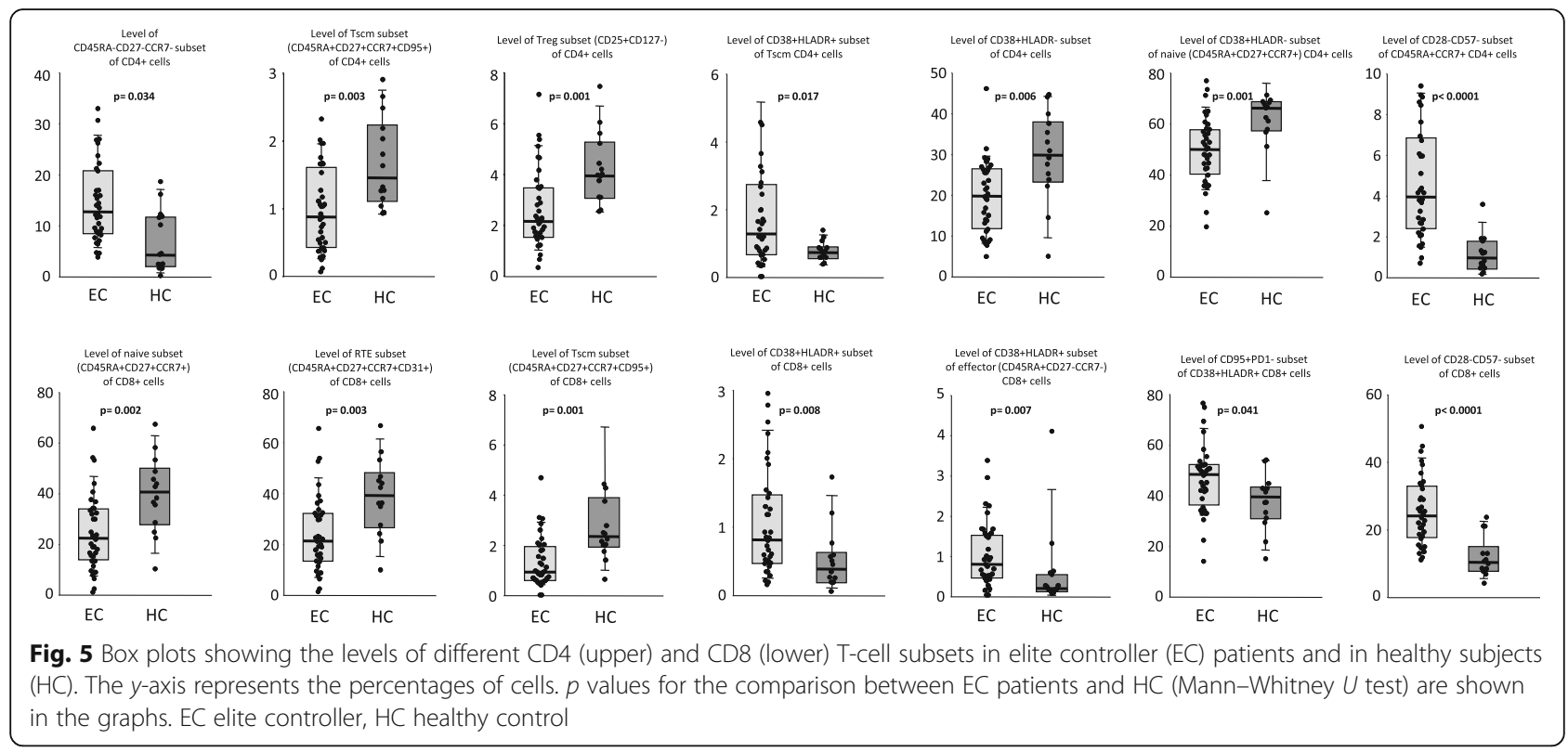

PLS model. The absolute value of the loading of each immunological variable in the PLS model is a measure of the weight of the variable in the model and thus, the higher the loading the higher the probability of significant differences between the two groups (EC cases and EC controls). In fact, for the entire set of 77 immunological variables selected by the PLS model, there was a significant inverse correlation between the absolute value of the loadings and the $p$ values obtained by the Mann-Whitney test (Additional file 6), meaning that the higher the absolute value of the loading, the higher the probability of a statistically significant $(p<0.05)$ difference.

Moreover, using the Mann-Whitney $U$ test, it was possible to evaluate those variables significantly different between EC cases and EC controls that were not selected by the PLS model due to a numerical effect. This approach allowed us to detect another nine immunological variables with levels significantly different between EC cases and EC controls. A complete list of immunological variables significantly different by the Mann-Whitney test between EC cases and EC controls is shown in Additional file 7.

Figure 6 shows levels for the most relevant variables for EC cases and EC controls. Among CD4 subsets, EC cases presented lower levels of CD38 expression on the effector (CD45RA + CD27-CCR7-) subset of CD4 cells. In contrast, the level of PD1 expression on total and on central memory (CD45RA-CD27 + CCR7+) CD4 cells was higher in EC cases compared to EC controls. Among CD8 T-cell subsets, compared to EC controls, EC cases showed lower levels of naïve (CD45RA + CD27 + CCR7+) and of

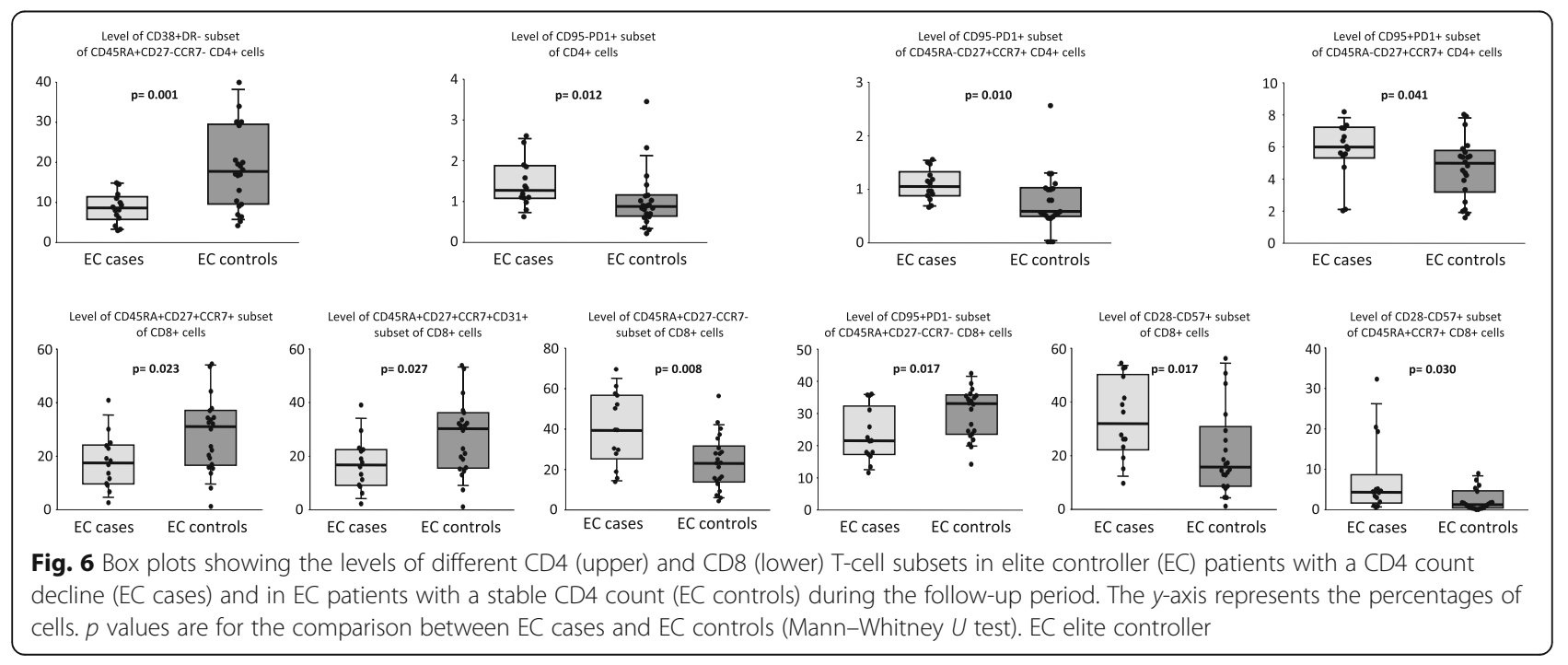


RTE (naïve cells expressing CD31) subsets. In contrast, the level of the effector (CD45RA + CD27-CCR7-) subset was higher in EC cases as was the level of senescence (CD28-CD57+) in total CD8 cells and in naive CD8 cells. Lastly, expression of CD95 in the effector subset of CD8 cells was lower in EC cases.

Overall, for those T-cell subsets that were significantly different in EC patients with respect to HCs, the levels for EC controls were intermediate between those found in EC cases and HCs (Fig. 7). Thus, levels in EC controls were more similar to levels in HCs than were levels in EC cases, and for some of these subsets, the levels for EC controls and $\mathrm{HCs}$ were not significantly different. This was the case for levels of the CD45RA-CD27CCR7- subset of CD4 cells and for the levels of CD28 + CD57- subset of CD8 cells (Fig. 7).

\section{Discussion}

The present study was designed to test the hypothesis that perturbations in immunological parameters related to Tcell homeostasis may be associated with CD4 T-cell loss in EC patients maintaining long-term undetectable levels of plasma HIV viremia. The main findings of our study are: (1) there is an important perturbation of T-cell homeostasis in EC patients despite long-term undetectable viremia; (2) based on the T-cell parameters analyzed, a PLS-CM statistical approach was able to discriminate and to predict, with high sensitivity and specificity, EC patients experiencing immunological progression from those EC patients with a stable CD4 count; and (3) immunological parameters associated with the loss of CD4 T cells in EC patients included mainly those related to $\mathrm{CD} 8 \mathrm{~T}$-cell homeostasis.
Despite the persistent control of HIV replication to undetectable levels, several alterations related to a state of systemic inflammation have been recognized in ECs compared to seronegative subjects and to HIV patients with cARTmediated control of HIV replication [23-25], and this has been associated with an increased rate of morbidity and mortality in EC patients [24-26]. Our study demonstrates that immunological alterations in EC patients are not limited to markers of systemic inflammation and extend to several cellular markers of T-cell homeostasis. Although the EC patients included in our study had maintained longterm undetectable plasma HIV viremia, they clearly departed from seronegative healthy subjects (HCs) regarding the distribution of T-cell subsets. As many as 93 immunological variables were necessary to discriminate between EC patients and HCs and many of these presented levels significantly different between ECs and HCs, suggesting there is an important perturbation of T-cell homeostasis in EC patients despite long-term undetectable viremia.

Different processes involved in T-cell homeostasis were altered in ECs compared to healthy subjects. Among them, naïve and RTE subsets, stem cell memory subsets, regulatory $\mathrm{T}$ cells, and expression of the costimulatory receptor CD28 were significantly decreased, whereas activation and expression of CD95 were significantly increased. Some of these perturbations have already been reported in different cohorts of ECs, such as alterations in naïve/memory subsets [17, 19], increased activation [15, 18, 27], increased senescence [27], and decreased regulatory T cells [28]. However, EC cohorts employed in these previous studies had varying degrees of HIV-associated immune suppression (as reflected in CD4 counts) and different cutoffs of plasma viremia, meaning that
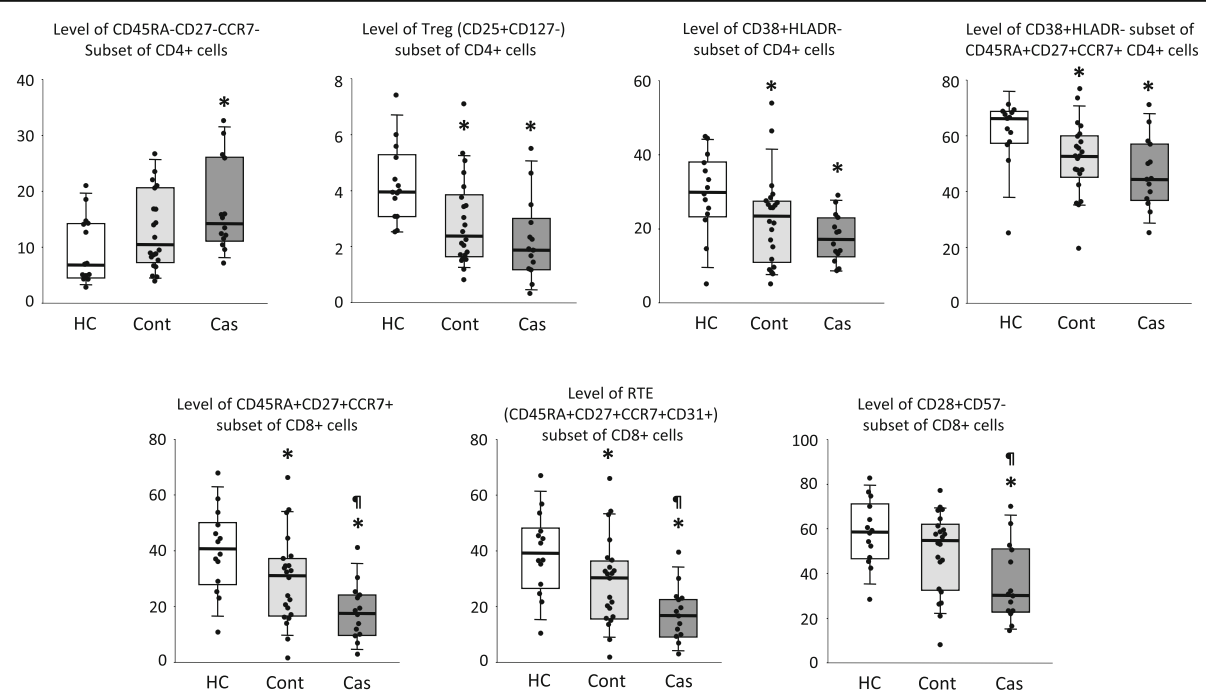

Fig. 7 Box plots showing the levels of different CD4 (upper) and CD8 (lower) T-cell subsets in healthy subjects (HCs), elite controller (EC) patients with a stable CD4 count (Cont), and in EC patients with a CD4 count decline (Cas) during the follow-up period. The $y$-axis represents the percentages of cells. Significant differences $(p<0.05)$ with respect to HCs are marked with an asterisk $\left(^{*}\right)$ and between the Cas and Cont groups of EC patients with the symbol. EC elite controller, Cas EC case, Cont EC control, HC healthy control 
some alterations reported in these studies could be influenced by CD4 counts [28] or low-level plasma HIV viremia [27]. Since all EC patients included in our study had high $\mathrm{CD} 4$ counts at the time of the immunophenotypic analysis, our results demonstrate that perturbation of T-cell homeostasis is already present in ECs from the early stages of HIV disease. Moreover, our study is the first reporting alterations in ECs of some important parameters of T-cell homeostasis such as Tscm cells $[29,30]$. Interestingly Tscm cells were diminished in both CD4 and CD8 T cells and thus, the potential to repopulate other subsets of memory cells [31] may be compromised in ECs at early stages of infection.

The most relevant findings of our study are those related to T-cell parameters associated with loss of immunological control in ECs. Using a set of 77 different immunological variables, the PLS model was able to discriminate, with high sensitivity and specificity, between EC patients showing a significant CD4 decline (cases) and those with stable CD4 counts (controls). The immunological variables were assessed when both groups of EC patients presented similar high levels of CD4 counts (prior to CD4 T-cell loss in EC cases) and thus, the differences found cannot be attributed to different degrees of HIV-associated immune suppression, but rather they are characteristic of some EC subjects and likely involved in the decline of CD4 T cells observed in EC cases. Since no functional methods were employed in this study, we can speculate only about the potential mechanisms involved in the differences observed in the extent of T-cell homeostasis disturbance between EC cases and EC controls. Besides viral factors, such as the higher levels of residual viral replication in EC cases [10], several host-related factors could contribute to the CD4 T-cell loss and T-cell homeostasis disturbance observed in EC cases. Among them are defective thymic function [17], lymphoid tissue fibrosis [32], genetic variation in different genes related to immune response $[33,34]$, and a cytokine-driven inflammatory milieu [35].

Interestingly, the two-sample non-parametric test (Mann-Whitney $U$ test) was not able to detect subtle but important differences between cases and controls for many of the immunological variables that were detected by the PLS model. PLS-CM is an approach that is best suited to extracting these differences employing latent factors and building a predictive model in situations with a small sample size and many predictive variables. In agreement with this higher sensitivity of PLS modeling to detect differences among a large panel of variables, the immune parameters that had significant differences by the two-sample test were those showing the highest loadings in the PLS model.

The factors involved in the immunological progression observed in some ECs that maintain virological control are largely unknown and studies addressing this issue are scarce, with only a few investigating potential associated immunological factors [10, 15, 36, 37]. In some of these studies, T-cell activation was shown to be associated with disease progression in ECs using a cross-sectional design $[15,36]$. The other two studies addressing the association of T-cell activation with CD4 evolution in EC patients and using a longitudinal follow-up period similar to our study $[10,37]$ found discordant results. One reported increased activation associated with immunologic progression [10] but the other did not [37]. Differences in the criteria used to define immunological progression may explain the discordant results. Moreover, both studies analyzed T-cell activation when CD4 counts were significantly different between EC patients showing immunological progression and those maintaining stable CD4 counts, which is in clear contrast with our study.

Our results show that several T-cell subsets showed significant differences between EC cases and controls and interestingly they were mainly related to CD8 cell homeostasis. Naïve and RTE subsets of CD8 cells were diminished in EC cases, suggesting either a defect in thymic output or an increased rate of transition of naïve cells to more differentiated subsets. Since a deficit in thymic output would be reflected in the RTE subset of both $\mathrm{CD} 8$ and $\mathrm{CD} 4$ compartments, our findings more strongly support an increased rate of differentiation. This, together with the finding of increased levels of effector CD8 cells in EC cases, supports the higher levels of residual HIV replication in EC cases. Unfortunately, we did not measure plasma HIV viremia with ultrasensitive assays and thus, we can only speculate on this possibility. However, in support of this hypothesis, previous studies have found higher levels of residual viremia in EC patients showing CD4 depletion [9, 10, 38]. Although levels of effector CD8 cells were higher in EC cases, CD8 $\mathrm{T}$ cells showed increased levels of the CD28-CD57 + subset, a phenotype that has been associated with replicative senescence [39]. CD8 T cells expressing this senescent phenotype have lower cytotoxic potential $[40,41]$ and produce high amounts of inflammatory cytokines such as IL6 and TNFo [39], promoting an inflammatory state that could contribute to the CD4 depletion observed in EC cases. An interesting finding was that naïve CD8 cells of EC cases also have increased levels of senescence, suggesting that they have undergone an antigen-independent expansion without differentiation to memory cells in an attempt to maintain CD8 T-cell homeostasis. Lastly, regarding alterations of CD4 T-cell subsets, EC cases had increased levels of expression of the cell exhaustion-associated marker PD1 in total CD4 cells and in central memory subset. Since overexpression of PD1 has been associated with a lower ability to reconstitute CD4 T cells in HIV patients with cART-mediated suppression of viral replication $[42,43]$, this increased 
expression of PD1 in EC cases could be another factor contributing to CD4 depletion.

Finally, there are some caveats that deserve comment. First, studies employing EC patients are very limited in sample size given the very low frequency of this exceptional population of individuals among the whole population of HIV-infected patients. However, in our study, we addressed the potential bias of a small sample size by studying a very homogeneous study population. Also, for the data analyses we employed the PLS regression, which is a statistical technique for performing multivariate calibration, especially when there are more variables than samples. Second, although the objective of our study had no such scope, it could have been interesting to include a group of HIV-normal progressors to assess whether the underlying mechanisms of the loss of immune control in EC patients are comparable to those underlying the non-immunological control in normal HIV+ progressors patients, as well as to evaluate the power of the PLS model to discriminate between both phenomena.

\section{Conclusions}

In summary, the present study is the first to perform an in-depth analysis of T-cell homeostasis disturbances in a well-characterized cohort of ECs with long-term control of HIV replication and showing different evolution of CD4 counts. We used a PLS-CM statistical approach that enabled us to detect subtle but important immunophenotype differences associated with the immunological progression. Overall, our results demonstrate the existence of an important perturbation of T-cell homeostasis in EC patients despite long-term undetectable viremia. For those EC patients experiencing immunological progression, those disturbances are mainly related to CD8 T-cell homeostasis. Our results may open new avenues for understanding the mechanisms underlying immunological progression despite HIV replication control, suggesting that active pathogenic mechanisms are still present in some EC patients. These findings should be considered to improve the clinical management of this exceptional group of patients, and eventually in seeking a functional cure through immune-based clinical trials.

\section{Additional files}

Additional file 1: Flow diagram showing the inclusion criteria and the sequential strategy for selecting the patients included in the study. Numbers inside the boxes indicate the number of patients selected after each step of the selection process. (DOC $200 \mathrm{~kb}$ )

Additional file 2: Monoclonal antibodies and fluorochromes used in the study. (DOC $49 \mathrm{~kb}$ )

Additional file 3: Staining conditions for immunophenotypic analysis and definitions of T-cell subsets analyzed. (DOC $29 \mathrm{~kb}$ )

Additional file 4: Partial least-squares-class modeling (PLS-CM). (DOC $33 \mathrm{~kb}$ )
Additional file 5: Immunological variables (in CD4 and CD8 T-cell subsets) with significant differences between EC patients and healthy subjects (HCs) (Mann-Whitney $U$ test). (DOC $119 \mathrm{~kb}$ )

Additional file 6: Scatterplot showing loading values (coefficients) for the 77 immunological variables selected by the PLS model versus $p$ values obtained when comparing levels of these variables between EC cases and EC controls with the Mann-Whitney $U$ test. The dotted line marks the threshold for statistical significance $(p<0.05$ ). (DOC $67 \mathrm{~kb}$ )

Additional file 7: Immunological variables (in CD4 and CD8 T-cell subsets) with significant differences between EC cases and EC controls (Mann-Whitney $U$ test). (DOC $52 \mathrm{~kb}$ )

Additional file 8: Clinical centers and research groups that contribute to ECRIS. (DOC $33 \mathrm{~kb}$ )

\section{Abbreviations}

AIDS: Acquired immune deficiency syndrome; CART: Combination antiretroviral therapy; EC: Elite controller; EDTA: Ethylenediamine tetraacetic acid; HC: Healthy control; HIV-1: Human immunodeficiency virus-1; LTNP: Long-term non-progressor; LV: Latent variable; PBMC: Peripheral blood mononuclear cell; PLS-CM: Partial least-squares-class modeling; pVL: Plasma viral load; RNA: Ribonucleic acid; RTE: Recent thymic emigrant;

SPSS: Statistical Package for the Social Sciences; Treg cell: T regulatory cell; Tscm cell: T memory stem cell; VIP: Variable influence on projection

\section{Acknowledgements}

We especially thank Noelia Rodriguez for her technical assistance. We want to particularly acknowledge the patients in this study for their participation and to the HIV BioBank, which is integrated into the Spanish AIDS Research Network (RIS), and collaborating centers for the generous gifts of clinical samples used in this work. This study would not have been possible without the collaboration of the medical, nursing, staff and data managers who have taken part in the project (see Additional file 8).

\section{Funding}

This work has been partially funded by projects RD12/0017/0031, RD16/0025/ 0013, and SAF2015-66193-R as part of the Health Research and Development Strategy, State Plan for Scientific and Technical Research and Innovation (20082011 and 2013-2016) and cofinanced by the Institute of Health Carlos III (ISCIII), Sub-Directorate General for Research Assessment and Promotion and European Regional Development Fund. NR is a Miguel Servet investigator from the ISCIII (CP14/00198), Madrid, Spain. C Restrepo was funded by project RD12/0017/ 0031 and is currently funded by project RD16/0025/0013. M García is a predoctoral student co-funded by grant CP14/00198 and an Intramural Research Scholarship from Instituto de Investigación Sanitaria-Fundación Jiménez Díaz (IIS-FJD). The funders had no role in study design, data collection and analysis, the decision to publish, or the preparation of the manuscript.

\section{Availability of data and materials}

The datasets supporting the conclusions of this article are included within the article (and its additional files).

\section{Authors' contributions}

$N R, J M B, A L, E R M, J A, M L, F G$, and MAMF conceived the study. NR and JMB designed the study and wrote the manuscript, which was reviewed by all authors. C Rodriguez, JdR, RP, M Górgolas, and AC contributed to recruitment and follow-up of patients. M Garcia and C Restrepo carried out the experimental work. JML and MM supervised the flow cytometry experiments. NR, JMB, MCO, and LAS undertook the statistical analysis. All authors read and approved the final manuscript.

Ethics approval and consent to participate

To participate in the study, written informed consent was obtained from all individuals. The study protocol was evaluated and approved by the institutional ethical committee of Carlos III Hospital with reference number HCIII-P3/14.

Consent for publication Not applicable. 


\section{Competing interests}

The authors declare that they have no competing interests.

\section{Publisher's Note}

Springer Nature remains neutral with regard to jurisdictional claims in published maps and institutional affiliations.

\section{Author details}

'IIS-Fundación Jiménez Díaz, UAM, Av. Reyes Católicos, 2, 28040 Madrid, Spain. ${ }^{2}$ Hospital Universitario Rey Juan Carlos, Móstoles, Madrid, Spain. ${ }^{3}$ Universidad de Burgos, Burgos, Spain. ${ }^{4}$ Hospital Clinic-IDIBAPS, HIVACAT, Universidad de Barcelona, Barcelona, Spain. ${ }^{5}$ Centro Nacional de Investigaciones Cardiovasculares, Madrid, Spain. ${ }^{6}$ Hospital Virgen del Rocío, Sevilla, Spain. ${ }^{7}$ Unidad de E. Infecciosas. Hospital Virgen de la Victoria e IBIMA, Málaga, Spain. ${ }^{8}$ Hospital Universitario Fundación Jiménez Díaz, Madrid, Spain. ${ }^{9}$ Centro Sanitario Sandoval, Instituto de Investigación Sanitaria del Hospital Clínico San Carlos (IdISSC), Madrid, Spain. ${ }^{10}$ Laboratory of Molecular Immuno-Biology, Hospital General Universitario Gregorio Marañón, Instituto de Investigación Sanitaria Gregorio Marañón, Madrid, Spain. ${ }^{11}$ AIDS Immunopathology Unit, Instituto de Salud Carlos III, Majadahonda, Madrid, Spain.

Received: 20 June 2017 Accepted: 13 February 2018

Published online: 28 February 2018

\section{References}

1. Sajadi MM, Constantine NT, Mann DL, Charurat M, Dadzan E, Kadlecik P, et al. Epidemiologic characteristics and natural history of HIV-1 natural viral suppressors. J Acquir Immune Defic Syndr. 2009;50:403-8.

2. Autran B, Descours B, Avettand-Fenoel V, Rouzioux C. Elite controllers as a model of functional cure. Curr Opin HIV and AIDS. 2011;6:181-7.

3. Cockerham LR, Hatano $\mathrm{H}$. Elite control of HIV: is this the right model for a functional cure? Trends Microbiol. 2015;23:71-5.

4. Shasha D, Walker BD. Lessons to be learned from natural control of HIV-future directions, therapeutic, and preventive implications. Front Immunol. 2013:4:1-8

5. Deeks SG, Walker BD. Human immunodeficiency virus controllers: mechanisms of durable virus control in the absence of antiretroviral therapy. Immunity. 2007;27:406-16.

6. Pereyra F, Addo M, Kaufmann DE, Liu Y, Miura T, Rathod A, et al. Genetic and immunologic heterogeneity among persons who control HIV infection in the absence of therapy. J Infect Dis. 2008;197:563-71.

7. Walker BD, Yu XG. Unravelling the mechanisms of durable control of HIV-1. Nat Rev Immunol. 2013;13:487-98.

8. Madec Y, Boufassa F, Porter K, Meyer L. CASCADE collaboration. Spontaneous control of viral load and CD4 cell count progression among HIV-1 seroconverters. AIDS. 2005:19:2001-7.

9. Boufassa F, Saez-Cirión A, Lechenadec J, Zucman D, Avettand-Fenoel V, Venet A, et al. CD4 dynamics over a 15 year-period among HIV controllers enrolled in the ANRS French observatory. PLoS One. 2011;6:e18726.

10. Noel N, Lerolle N, Lecuroux C, Goujard C, Venet A, Saez-Cirión A, et al. Immunologic and virologic progression in HIV controllers: the role of viral blips and immune activation in the ANRS CO21 CODEX study. PLoS One. 2015;10:e0131922.

11. Leon A, Perez I, Ruiz-Mateos E, Benito JM, Leal M, Lopez-Galindez C, et al. Rate and predictors of progression in elite and viremic HIV-1 controllers. AIDS. 2016;30:1209-20.

12. Okoye AA, Picker LJ. CD4(+) T-cell depletion in HIV infection: mechanisms of immunological failure. Immunol Rev. 2013;254:54-64.

13. Paiardini M, Muller-Trutwin M. HIV-associated chronic immune activation. Immunol Rev. 2013;254:78-101.

14. Moir S, Chun TW, Fauci AS. Pathogenic mechanisms of HIV disease. Annu Rev Pathol. 2011;6:223-48.

15. Hunt PW, Brenchey J, Sinclair E, McCune JM, Roland M, Page-Shafer K, et al. Relationship between T cell activation and CD4 T cell count in VIVseropositive individuals with undetectable plasma HIV RNA levels in the absence of therapy. J Infect Dis. 2008;197:126-33.

16. López M, Soriano V, Peris-Pertusa A, Rallón N, Benito JM. Elite controllers display higher activation on central memory CD8 T cells than HIV patients successfully on HAART. AIDS Res Hum Retrovir. 2011;27:157-65.
17. Yang Y, Al-Mozaini M, Buzón MJ, Beamon J, Ferrando-Martínez S, Ruiz-

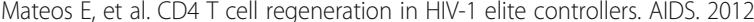
26:701-6.

18. Sajadi MM, Pulijala R, Redfield RR, Talwani R. Chronic immune activation and decreased CD4 counts associated with hepatitis C infection in HIV-1 natural viral suppressors. AIDS. 2012;26:1879-84.

19. Sajadi MM, Redfield RR, Talwani R. Altered T-cell subsets in HIV-1 natural viral suppressors (elite controllers) with hepatitis C infection. AIDS. 2013;27:1989-95.

20. Ramirez CM, Sinclair E, Epling L, Lee SA, Jain V, Hsue PY, et al. Immunologic profiles distinguish aviremic HIV-infected adults. AIDS. 2016;30:1553-62.

21. Sobrino-Vegas $P$, Gutiérrez F, Berenguer J, Labarga $P$, et al. [The Cohort of the Spanish HIV Research Network (CoRIS) and its associated biobank; organizational issues, main findings and losses to follow-up.] In Spanish. Enferm Infecc Microbiol Clin. 2011;29:645-53.

22. García-Merino I, de Las CN, Jiménez JL, Gallego J, Gómez C, Prieto C, et al. Spanish HIV BioBank. The Spanish HIV BioBank: a model of cooperative HIV research. Retrovirology. 2009;6:27.

23. Krishnan S, Wilson EM, Sheikh V, Rupert A, Mendoza D, Yang J, et al Evidence for innate immune system activation in HIV type-1 elite controllers. J Infect Dis. 2014;209:931-9.

24. Pereyra F, Lo J, Triant V, Wei A, Buzon M, Fitch K, et al. Increased coronary atherosclerosis and immune activation in HIV-1 elite controllers. AIDS. 2012; 26:2409-12.

25. Hsue PY, Hunt PW, Schnell A, Kalapus SC, Hoh R, Ganz P, et al. Role of viral replication, antiretroviral therapy and immunodeficiency in HIV-associated atherosclerosis. AIDS. 2009;23:1059-67.

26. Crowell TA, Gebo KA, Blankson JN, Korthuis PT, Yehia BR, Rutsein RM, et al, Hospitalization rates and reasons among HIV elite controllers and persons with medically controlled HIV infection. J Infect Dis. 2012;211:1692-702.

27. Ruiz-Mateos E, Ferrando-Martínez S, Machmach K, Viciana P, Pacheco YM, Nogales N, et al. High levels of CD57+CD28- T-cells, low T-cell proliferation and preferential expansion of terminally differentiated CD4+ T-cells in HIVelite controllers. Curr HIV Res. 2010;8:471-81.

28. Hunt PW, Landay AL, Sinclair E, Martinson JA, Hatano H, Emu B, et al. A low regulatory cell response may contribute to both viral control and generalized immune activation in HIV controllers. PLoS One. 2011;6:e15924.

29. Gattinoni L, Speiser DE, Lichterfeld M, Bonini C. T memory cells in health and disease. Nat Med. 2017:23:18-27.

30. Chahroudi A, Silvestri G, Lichterfeld M. T memory stem cells and HIV: a long term relationship. Curr HIV/AIDS Rep. 2015:12:33-40.

31. Gattinoni L, Lugli E, Ji Y, Pos Z, Paulos CM, Quigkey MF, et al. A human memory T cell subset with stem cell-like properties. Nat Med. 2011;17:1290-7.

32. Sanchez JL, Hunt PW, Reilly CS, Hatano H, Beilman GJ, Khoruts A, et al. Lymphoid fibrosis occurs in long-term nonprogressors and persists with antiretroviral therapy but may be reversible with curative interventions. J Infect Dis. 2015;211:1068-75.

33. Dominguez-Molina B, Tarancón-Diez L, Hua S, Abad-Molina C, RodriguezGallego E, Machmach K, et al. HLA-B*57 and IFNL4-related polymorphisms are associated with protection against HIV-1 disease progression in controllers. Clin Infect Dis. 2017;64:321-8.

34. Westrop SJ, Cocker ATH, Boasso A, Sullivan AK, Nelson MR, Imami N. Enrichment of HLA types and single-nucleotide polymorphism associated with non-progression in a strictly defined cohort of HIV-1 controllers. Front Immunol. 2017:8:746.

35. Li JZ, Arnold KB, Lo J, Dugast AS, Plants J, Ribaudo HJ, et al. Differential levels of soluble inflammatory markers by human immunodeficiency virus controller status and demographics. Open Forum Infect Dis. 2015;2:ofu117.

36. Bansal A, Sterret S, Erdman N, Westfall AO, Dionne-Odom J, Overton ET, et al. Normal T-cell activation in elite controllers with preserved CD4+ T-cell counts. AIDS. 2015:29:2245-54

37. Kamya P, Tsoukas CM, Boulet S, Routy JP, Thomas R, Côte P, et al. T cell activation does not drive CD4 decline in longitudinally followed HIVinfected elite controllers. AIDS Res Ther. 2011;8:20-6.

38. Pereyra F, Palmer S, Miura T, Block BL, Wiegand A, Rothchild AC, et al. Persistent low-level viremia in HIV-1 elite controllers and relationship to immunologic parameters. J Infect Dis. 2009;200:984-90.

39. Dock J, Effros RB. Role of CD8 T cell replicative senescence in human aging and in HIV-mediated immunosenescence. Aging Dis. 2011:2:382-97.

40. Dagarag $\mathrm{M}, \mathrm{Ng} \mathrm{H}$, Lubong R, Effros RB, Yang OO. Differential impairment of lytic and cytokine functions in senescent human immunodeficiency virus type 1-specific cytotoxic T lymphocytes. J Virol. 2003;77:3077-83. 
41. Yang OO, Lin $\mathrm{H}$, Dagarag $\mathrm{M}, \mathrm{Ng} H \mathrm{~L}$, Effros RB, Uittenbogaart $\mathrm{CH}$. Decreased perforin and granzyme B expression in senescent HIV-1-specific cytotoxic T lymphocytes. Virology. 2005;332:16-9.

42. Grabmeier-Pfistershammer K, Steinberger P, Rieger A, Leitner J, Kohrgruber N. Identification of PD-1 as a unique marker for failing immune reconstitution in HIV-1-infected patients on treatment. J Acquir Immune Defic Syndr. 2011;56: 118-24.

43. Nakanjako D, Ssewanyana I, Mayanja-Kizza H, Kiragga A, Colebunders R, Manabe YC, et al. High T-cell immune activation and immune exhaustion among individuals with suboptimal CD4 recovery after 4 years of antiretroviral therapy in an African cohort. BMC Infect Dis. 2011;11:43.

Submit your next manuscript to BioMed Central and we will help you at every step:

- We accept pre-submission inquiries

- Our selector tool helps you to find the most relevant journal

- We provide round the clock customer support

- Convenient online submission

- Thorough peer review

- Inclusion in PubMed and all major indexing services

- Maximum visibility for your research

Submit your manuscript at www.biomedcentral.com/submit 